JOURNAL OF THEORETICAL

AND APPLIED MECHANICS

56, 4, pp. 1083-1095, Warsaw 2018

DOI: $10.15632 /$ jtam-pl.56.4.1083

\title{
NUMERICAL LOSS ANALYSIS IN A COMPRESSOR CASCADE WITH LEADING EDGE TUBERCLES
}

\author{
Tan Zheng, XiaO-Qing Qiang, Jin-Fang Teng, Jin-Zhang Feng \\ School of Aeronautics and Astronautics, Shanghai Jiao Tong University, Shanghai, China \\ e-mail: qiangxiaoqing@sjtu.edu.cn
}

\begin{abstract}
A numerical analysis of loss has been carried out to explore the loss mechanism of leading edge tubercles in a high speed compressor cascade. Taking the lead from flippers of the humpback whale, tubercles are passive structures of a blade for flow control. Evaluation of the overall performance in terms of entropy increase shows that the loss reduction is achieved both at high negative and high positive incidence angles, while a rise in the loss is obtained near the design point. And a smaller wave number as well as a smaller amplitude results in lower additional losses at the design point. Spanwise and streamwise distributions of pitchwise-averaged entropy increase combined with flow details have been presented to survey the loss development and, subsequently, to interpret the loss mechanism. The tubercle geometry results in the deflection flow and the consequent spanwise pressure gradient. This pressure gradient induces formation of counter-rotating streamwise vortices, transports away the low-momentum fluid near wall from crests towards troughs and leads to local high loss regions behind troughs as well as loss reduction behind the crests in comparison to the baseline. The interaction between these vortices and flow separation by momentum transfer leads to separation delay and the consequent loss reduction at the outlet.
\end{abstract}

Keywords: compressor cascade, flow separation, passive flow control, leading edge tubercles, streamwise vortices, loss analysis

\section{Nomenclature}

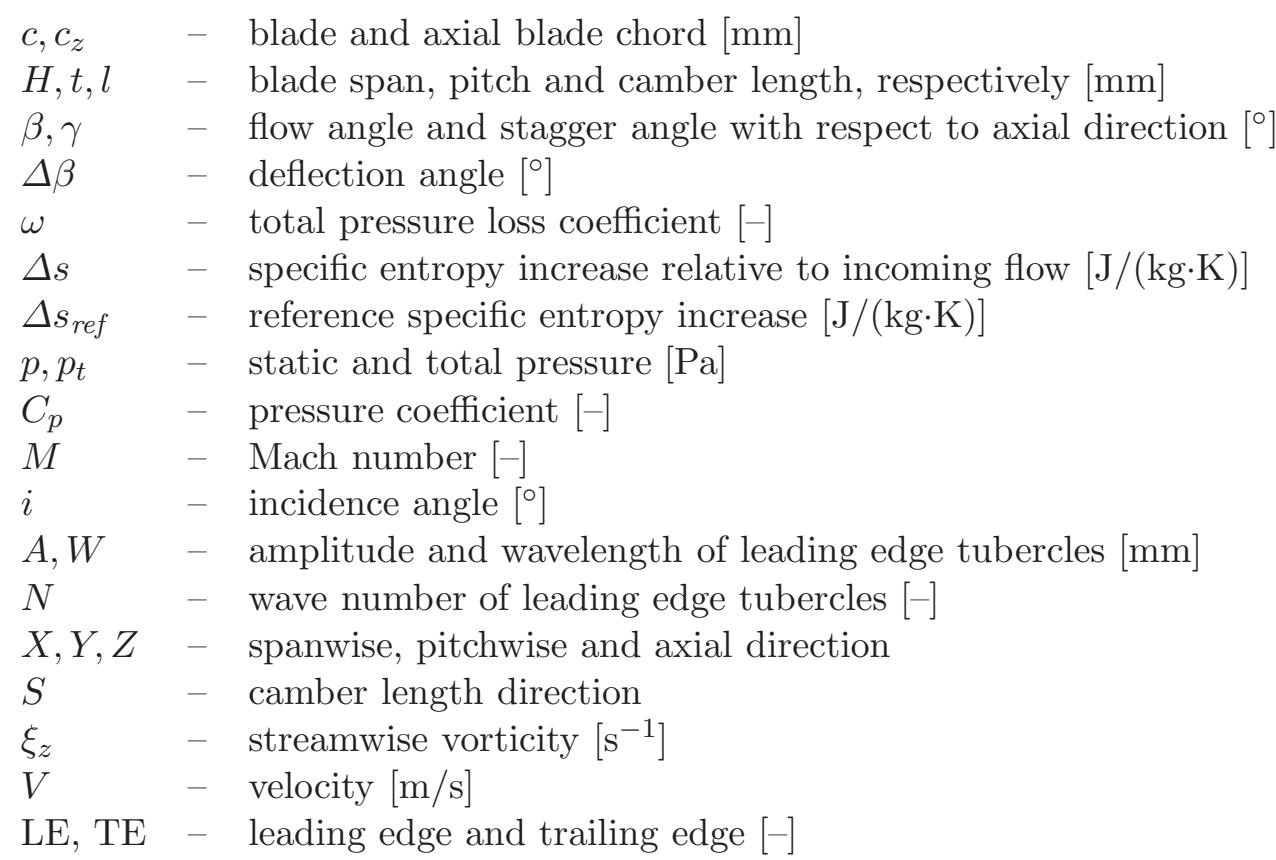




\section{Introduction}

Flow separation is a common phenomenon in the axial compressors that occurs near the blades. Severe separation contributes greatly to passage blockage and to aerodynamic losses. Worse yet, compressor stall may be induced, which subsequently leads to a sharp decrease in the aerodynamic performance.

Studies of active and passive flow control have been carried out with the aim of depressing flow separation. An overview of possible flow control methods in gas turbine engines is given by several researchers (Lord et al., 2000). Active flow control techniques have the advantage of being suitable for a wide range of operation conditions. Due to their increased complexity, however, gas turbine manufacturers are not fond of them as much as passive flow control techniques such as bowed stators (Fischer et al., 2003), non-axisymmetric profiled endwalls (Dorfner et al., 2011), vortex generators (Hergt et al., 2013). Another way for passive flow control is application of leading edge tubercles. This method is inspired by previous works on the morphology of humpback whale flippers by marine biologists (Fish and Battle, 1995).

A number of previous experimental and numerical works have been performed to explore the effects of leading edge tubercles on the performance of humpback whale flippers and isolated airfoils. A numerical study for the NACA 634-021 wing with and without tubercles shows that large streamwise vortices are formed in the regions posterior to the troughs between tubercles (Fish and Lauder, 2006). Johari et al. (2007) also take the NACA 634-021 airfoil as the baseline and carry out an experimental investigation comparing the effect of varying the wavelength and amplitude of sinusoidal tubercles on airfoil performance. Miklosovic et al. (2004) constructed two idealized scale models of a humpback pectoral flipper with and without leading edge tubercles and conducted wind-tunnel tests on them at $\mathrm{Re}=505000-520000$. The results showed that tubercles delayed the stall angle by approximately $40 \%$ while decreasing drag and increasing lift in the post-stall regime. Pedro and Kobayashi (2008) performed a numerical simulation of the setup used for the experimental studies (Miklosovic et al., 2004) with the detached eddy turbulence model. They indicated that the higher aerodynamic performance for the scalloped flipper results from the presence of streamwise vortices originated by the tubercles (Pedro and Kobayashi, 2008).

Also, leading edge tubercles have been applied in cascades. An experimental investigation (Keerthi et al., 2014) was carried out to quantify aerodynamic benefits of sinusoidal tubercles in a linear compressor cascade with different tubercle configurations at low Reynolds numbers $(\operatorname{Re}=130000)$. The results indicated that the performance of the cascade had substantially improved due to the effect of tubercles in terms of delaying the stall angle.

In consideration of the fact that the application of leading edge tubercles is not widespread in turbomachinery, the present work is committed to clarifying the effects of leading edge tubercles on the performance of a high-speed linear compressor cascade and elaborating the underlying loss mechanism of tubercle structures. The overall performance in terms of entropy increase is evaluated over the operating range of the cascade with different tubercle configurations. Spanwise and streamwise entropy distributions are utilized to investigate the difference in the loss development between the cascades with and without leading edge tubercles. Entropy distributions with flow visualizations on several axial cross-sections are examined in detail to interpret the loss development procedure.

\section{Numerical setup}

\subsection{Baseline cascade}

A high-speed linear compressor cascade is used in this study. The blade profile of the baseline cascade is modified from an airfoil of a high loading stator of a compressor. The airfoil is suitable 
to be used in axial compressors with a subsonic inlet flow over the full blade span. The blade profile and geometrical definitions are shown in Fig. 1.

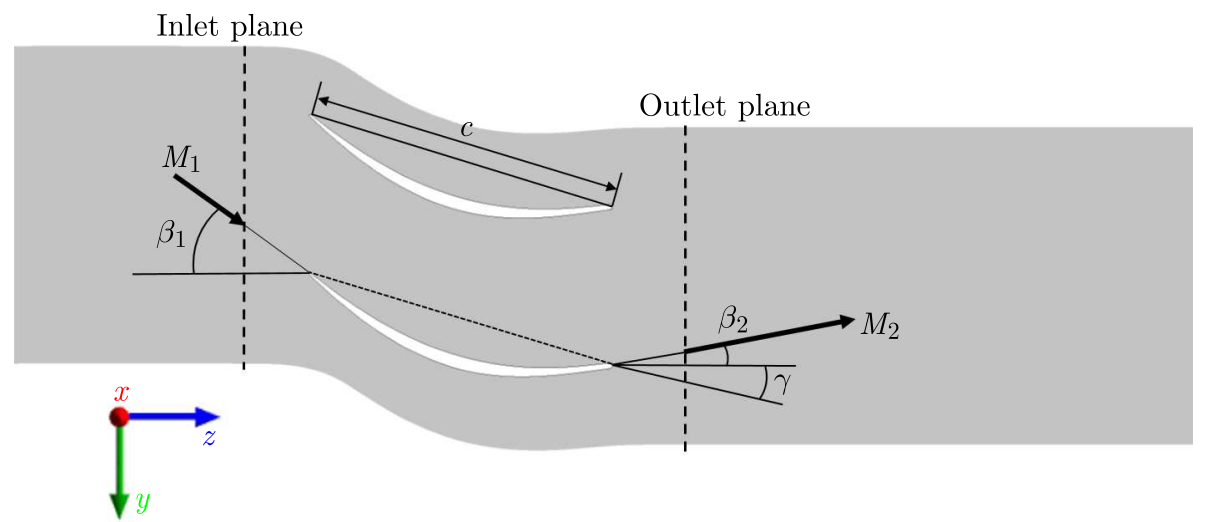

Fig. 1. Blade profile and geometrical definitions

The general design parameters of the cascade are listed in Table 1 . The cascade is built with a blade aspect ratio of 0.48 and a design inlet Mach number of 0.64 . This aspect ratio is selected in order to study the effects of leading edge tubercles in a certain blade span of about $50 \%$ chord length. Furthermore, this value is also related to the number and wavelength of tubercles. Based on the design inflow angle $\left(\beta_{1}=42^{\circ}\right)$, numerical simulations are performed in a wide operation range of incidence angles from $-16^{\circ}$ to $16^{\circ}$.

Table 1. General design parameters of the cascade

\begin{tabular}{|c|c|c|c|c|c|c|c|c|c|}
\hline$M_{1}$ & $\beta_{1}$ & $\beta_{2}$ & $\gamma$ & $\Delta \beta$ & $H$ & $c$ & $l$ & $H / c$ & $c / t$ \\
\hline \hline 0.64 & $42^{\circ}$ & $-9^{\circ}$ & $16.9^{\circ}$ & $51^{\circ}$ & $24.5 \mathrm{~mm}$ & $51 \mathrm{~mm}$ & $53.2 \mathrm{~mm}$ & 0.48 & 2 \\
\hline
\end{tabular}

\subsection{Leading edge tubercles}

The structure of tubercles is built by using a sinusoidal-like curve as the leading edge. The characteristic geometry definitions of tubercles are shown in Fig. 2a. What calls for special attention is that the horizontal axis $S$ represents the camber length direction and the longitudinal axis $X$ means the spanwise direction. The amplitude $A$ and wavelength $W$, characteristic dimen-

(a)

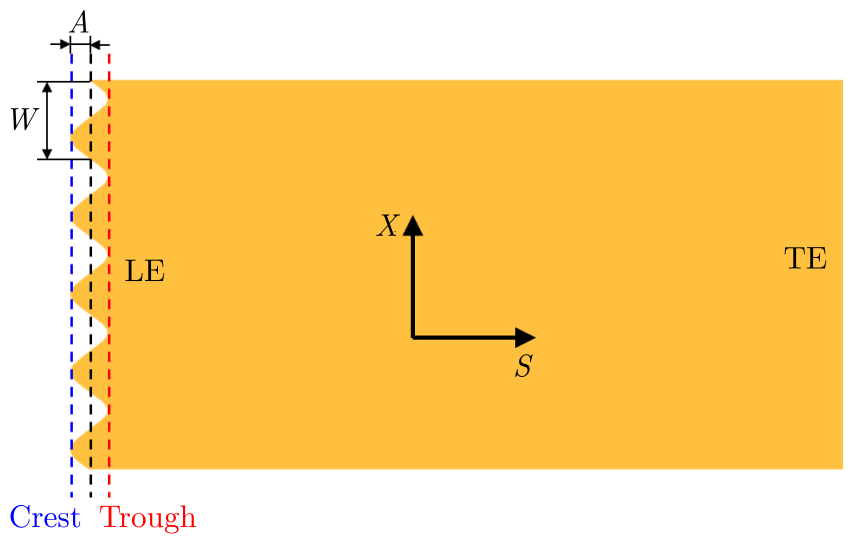

(b)

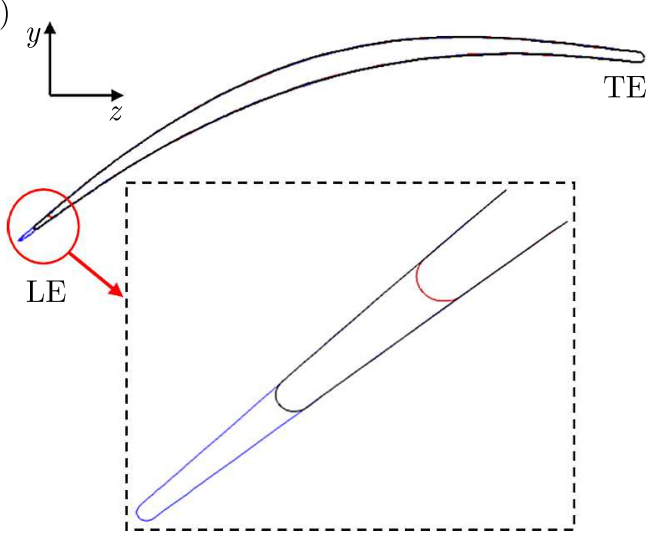

Fig. 2. (a) Characteristic geometry definitions of tubercles. (b) A sketch of profiles for the crest, middle and trough cross-sections 
sions of the sinusoidal-like curve are selected to study their effects on the compressor cascade performance.

In order to have a distinct comparison of the baseline geometry and two modified ones, a sketch of three blade profiles is shown in Fig. 2b. They have the same shape despite the difference in the leading edge.

Different computational cascade models are constructed covering variations in the amplitude and wavelength (or wave number). Different amplitudes of $A=0.02 l, 0.031$ and $0.04 \mathrm{l}$ (named as $A_{2}, A_{3}$ and $A_{4}$ ) combined with several wavelengths of $W=0.16 c, 0.12 c$ and $0.096 c$ (or several wave numbers of $N=3,4$ and 5) are systematically investigated in this work. These parameter values are selected according to the suggestions given by studies of pioneering researchers (Johari et al., 2007; Hansen et al., 2011). Table 2 shows notations of various configurations. According to the table, nine cascades are named by combining $A_{2}-A_{4}$ with $N_{3}-N_{5}$. Besides, ORI represents the baseline cascade.

Table 2. Notations of different configurations

\begin{tabular}{|l|c|c|c|c|c|c|}
\hline \multicolumn{1}{|c|}{ Notations } & $A_{2}$ & $A_{3}$ & $A_{4}$ & $N_{3}$ & $N_{4}$ & $N_{5}$ \\
\hline \hline Amplitude & $0.02 \mathrm{l}$ & $0.03 \mathrm{l}$ & $0.04 \mathrm{l}$ & - & - & - \\
\hline Wavelength & - & - & - & $0.16 c$ & $0.12 c$ & $0.096 c$ \\
\hline Wave Number & - & - & - & 3 & 4 & 5 \\
\hline
\end{tabular}

\subsection{Numerical method}

ANSYS-CFX software package has been used to perform RANS simulations on the baseline cascade and on the cascade with different leading edge tubercles. The overall performance of these cascades was investigated with the S-A turbulence model. As one of the most prominent turbulence models, the S-A model is widely used in turbomachinery due to its numerical efficiency and robustness. The Reynolds number based on the characteristic chord length and inlet velocity was about $7.9 \cdot 10^{5}$.

In the simulations of these cascades, fine multiblock structured grids with an H-O-H topology were constructed. The grid consisted of 3.1 million nodes with 97 pitchwise and 69 spanwise. Thus, the blade boundary layer was sufficiently resolved, where $y^{+} \approx 1$ was realized.

(a)

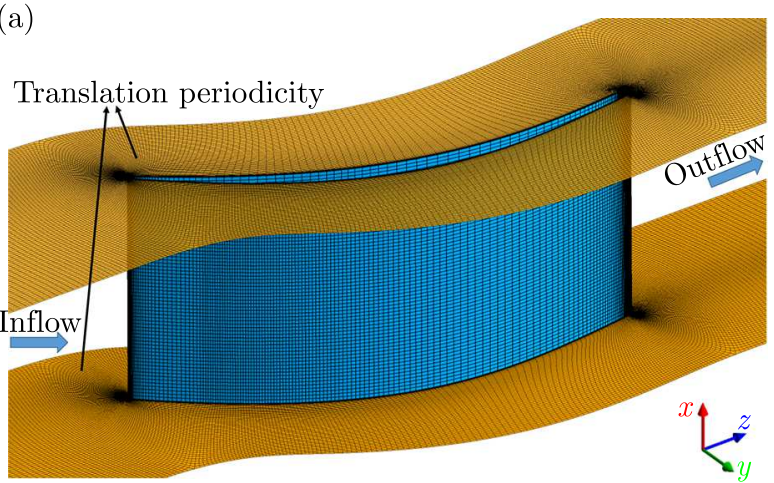

(b)

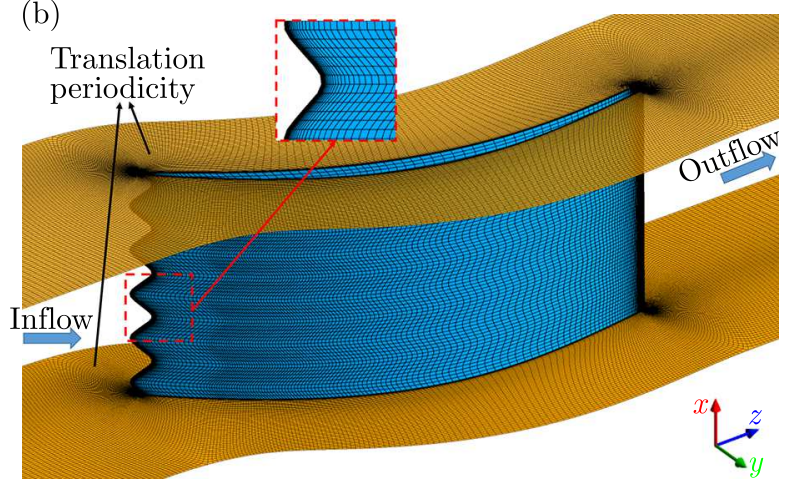

Fig. 3. Schematic diagram of cascade grids with and without leading edge tubercles;

(a) baseline cascade, (b) cascade with tubercles

Figure 3 shows a schematic diagram of the baseline cascade and the cascade with leading edge tubercles. At the inlet of the domain, boundary conditions consisted of uniform total pressure, total temperature and flow direction. The turbulence intensity of $5 \%$ was selected. At the outlet boundary, an average static pressure of $101325 \mathrm{~Pa}$ was imposed. Solid boundaries were applied 
at the blade surfaces with no slip and impermeability conditions. The side boundaries of the passage were modeled with matching translational periodicity. At the passage endwalls, the translational periodicity was also used so as to obtain an "infinite blade cascade".

A linear cascade constructed with the controlled diffusion airfoil (Steinert et al., 1991) was simulated to validate the numerical method mentioned above. The loading and flow phenomenon in this cascade was similar to the investigated cascade in the paper. Figure 4 shows experimental and numerical results of Mach number characteristics at the design point. The parameter $\bar{\omega}$ is calculated by following equations

$$
\bar{p}_{t}=\frac{\sum \dot{m} p_{t}}{\sum \dot{m}} \quad \bar{\omega}=\frac{\bar{p}_{t 1}-\bar{p}_{t 2}}{\bar{p}_{t 1}-\bar{p}_{1}}
$$

where $\bar{p}_{t}$ means the mass flow averaged total pressure in a local plane. The predicted results reasonably agree with the experimental data. This gives some confidence on the CFD model used in the following study.

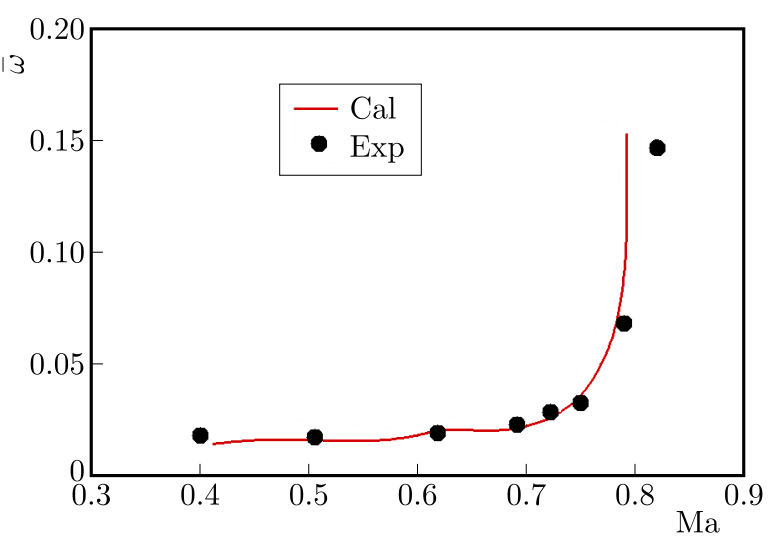

Fig. 4. Comparison of Mach number characteristics of experimental and numerical results

\section{Results and discussion}

\subsection{Overall loss characteristics}

For adiabatic flow through a stationary blade row, the total temperature is constant, and so entropy changes depend only on total pressure. Hence, for stator blades and cascade flows, total pressure losses can be taken to be synonymous with specific entropy increase (Denton, 1993).

In order to compare the overall loss characteristics of cascades with and without leading edge tubercles, the variation of the mass flow averaged specific entropy increase relative to incoming flow $\Delta s$ in the outlet plane is described for different wave numbers and amplitudes. The entropy increase is calculated and normalized with the reference value $\Delta s_{r e f}$, the entropy value in the outlet plane at the incidence angle of $i=0^{\circ}$.

In Fig. 5, the normalized entropy increase $\Delta s / \Delta s_{r e f}$ in the outlet plane is plotted against incidence angles for all the cascades. It is seen that the normalized entropy increase for the baseline cascade equals to 1 at the incidence angle of $0^{\circ}$. The minimum entropy increase of the baseline cascade is obtained at the incidence angle of $-4^{\circ}$. All the modified cascades have the same minimum loss incidence angle. Figure 5a shows the loss characteristics for cascades with a wave amplitude of 0.021 and different wave numbers of 3,4 and 5. For $A_{2} N_{3}, A_{2} N_{4}$ and $A_{2} N_{5}$ cascades, it is observed that the loss reduction is achieved in the range from $-16^{\circ}$ to $-8^{\circ}$ and from $8^{\circ}$ to $16^{\circ}$, compared to the baseline. However, in the operation conditions from $-8^{\circ}$ to $4^{\circ}$ incidence angles, the leading edge tubercles result in no loss reduction. In high negative incidence angles ranged from $-16^{\circ}$ to $-8^{\circ}$, the loss reduction gets larger as the wave number increases. 
And yet in high positive incidence angles ranged from $8^{\circ}$ to $16^{\circ}$, no definite trend in the loss reduction is observed as the wave number increases. Figure 5b shows the loss characteristics for $A_{2} N_{5}, A_{3} N_{5}$ and $A_{4} N_{5}$ cascades, with a wave number of 5 and different wave amplitudes of $0.021,0.031$ and 0.041 . In high negative incidence angles of $-16^{\circ}$ and $-12^{\circ}$, it is shown that the loss reduction, compared to the baseline, gets larger as the wave amplitude increases. The same relation between the loss reduction and wave amplitude is observed in the high positive incidence angles of $12^{\circ}$ and $16^{\circ}$. However, $A_{2} N_{5}, A_{3} N_{5}$ and $A_{4} N_{5}$ cascades show similar loss reductions as the wave amplitude increases at the incidence angle of $i=8^{\circ}$.

(a)

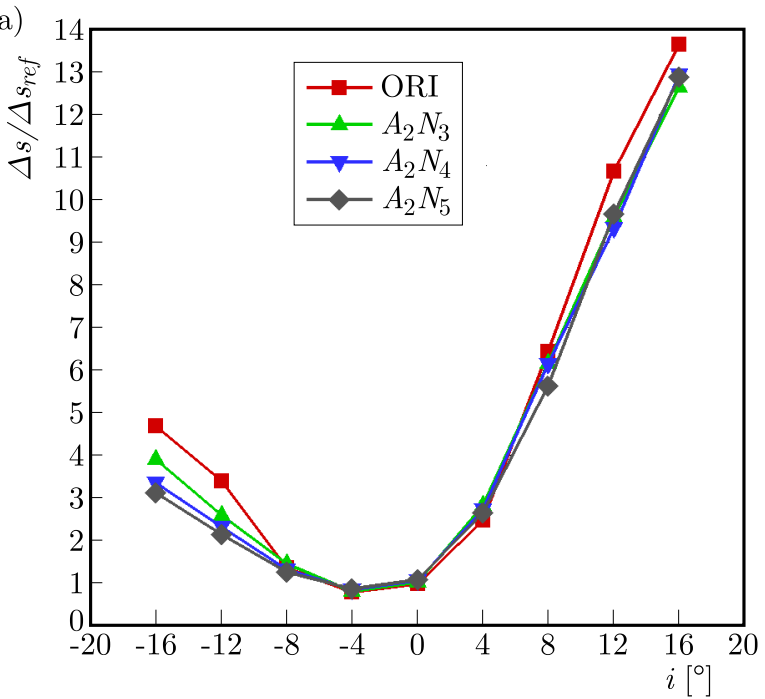

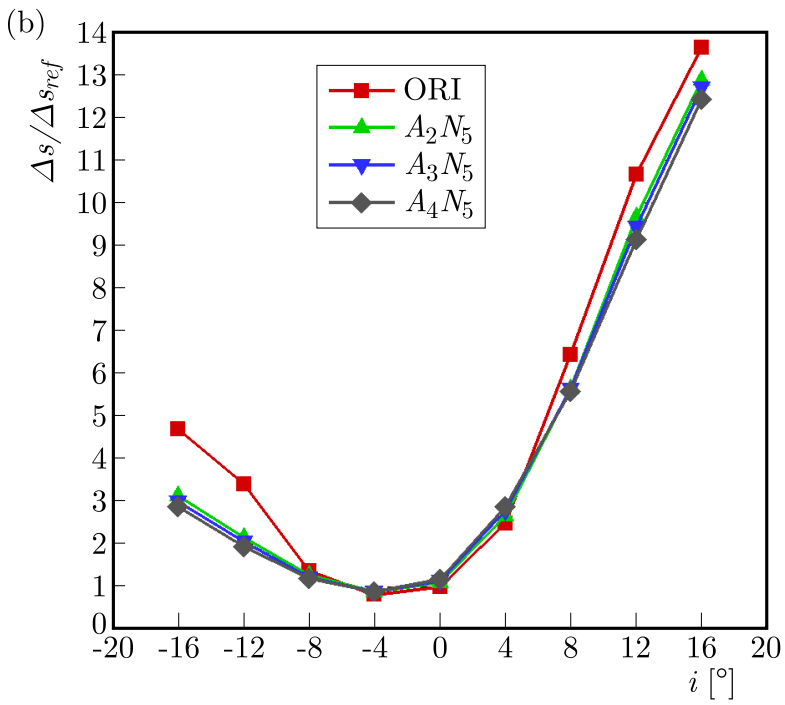

Fig. 5. Normalized entropy increase variation with incidence angle: (a) different wave numbers, (b) different wave amplitudes
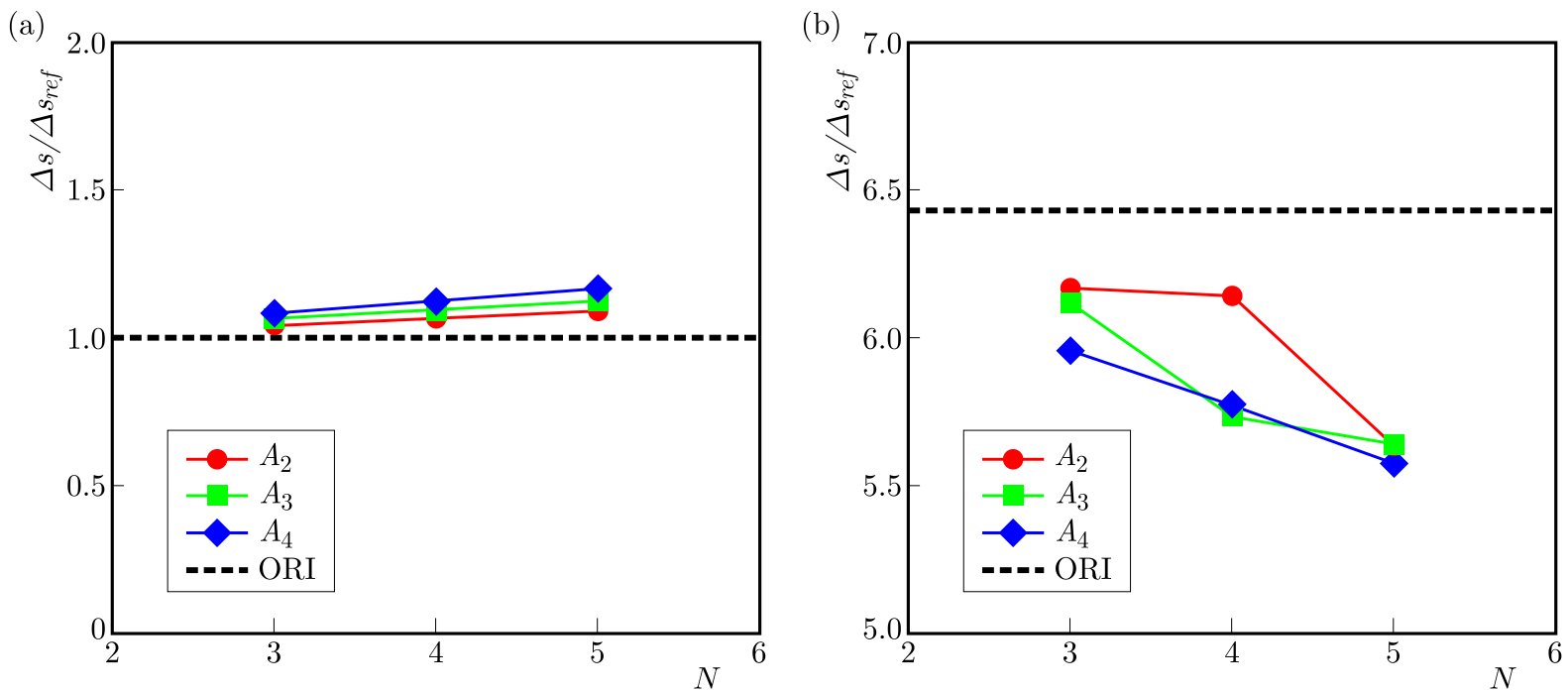

Fig. 6. Cascade loss characteristics for different wave numbers and amplitudes: (a) $i=0^{\circ}$, (b) $i=8^{\circ}$

In order to further explore the influence of tubercles on losses, Fig. 6 shows the results of normalized entropy increase predicted at two incidence angles of $i=0^{\circ}$ and $8^{\circ}$ for all the cascades. Compared to the baseline, a rise in the entropy increase is observed for all the tubercle configurations at $i=0^{\circ}$, just as shown in Fig. 6a. A smaller amplitude is shown to result in lower additional losses. It means that among all these amplitude configurations, the $A_{2}$ configuration with an amplitude of 0.021 produces the least negative influence on the cascade performance. 
Also, there exists a positive relation between the loss and wave number, which indicates a higher loss for a larger wave number, namely a smaller wavelength. For $A_{2} N_{5}$, A3N5 and A4N5 configurations, the losses respectively rise by $4.2 \%, 6.7 \%$ and $8.3 \%$ compared to the baseline. Figure $6 \mathrm{~b}$ shows that all cascades with leading edge tubercles obtain a decrease in the loss at $i=8^{\circ}$. And the loss reduction gets larger as the wave number increases. The normalized entropy increase equals to 6.4333 in the baseline configuration. The loss reductions for $A_{2} N_{5}, \mathrm{~A} 3 \mathrm{~N} 5$ and A4N5 configurations, respectively, equal to $12.4 \%, 12.3 \%$ and $13.3 \%$ in contrast to the baseline. These values are close to each other.

\subsection{Spanwise and streamwise entropy distributions}

In this Section, spanwise and stremwise distributions of pitchwise-averaged entropy increase are analyzed to survey the loss development in the baseline and $A_{2} N_{5}$ cascades.

Spanwise distributions of the normalized entropy increase at axial positions of $z / c_{z}=30 \%$, $90 \%$ and $150 \%$ are shown in Fig. 7. It is noted that the axial positions of $z / c_{z}=0 \%, 100 \%$ and $150 \%$ represent the leading edge, trailing edge and outlet. Three wave periods are included in the range from $20 \%$ to $80 \%$ span for $A_{2} N_{5}$. It is shown that the normalized entropy increase has little change along the span for ORI both at $i=0^{\circ}$ and $8^{\circ}$. For $A_{2} N_{5}$, a periodical variation of the entropy increase along the span is observed at both operating incidence angles. We focus on one of the periods in the range from $40 \%$ to $60 \%$ span for further analysis. The locations of $M_{0}$, trough, middle and crest sections are shown in Fig. 8. In Fig. 7a, a rise in the loss is observed in the range from $52 \%$ to $58 \%$ span for $A_{2} N_{5}$ compared to ORI at the axial position of $z / c_{z}=30 \%$, and the maximum rise of loss locates at the trough section. At $z / c_{z}=90 \%$, the loss reduction is almost not observed over the full span for $A_{2} N_{5}$. At $z / c_{z}=150 \%$, there exists loss reduction merely in a small range from $54 \%$ to $56 \%$ for $A_{2} N_{5}$, and the maximum loss reduction also locates at the trough section. In Fig. $7 \mathrm{~b}$, it is shown that there exists a reduction in the loss between $43 \%$ and $47 \%$ span at $z / c_{z}=30 \%$ for $A_{2} N_{5}$, compared to ORI. And the maximum loss reduction locates at the crest section at $i=8^{\circ}$. At $z / c_{z}=90 \%$, no loss reduction is achieved over the whole span for $A_{2} N_{5}$. However, at $z / c_{z}=150 \%$, the loss reduction is achieved over the whole span. It is seen that the spanwise variance of $\Delta s$ tends to be smaller along the streamwise direction for $A_{2} N_{5}$ and, finally, an approximately uniform distribution of $\Delta s$ is achieved.

(a)

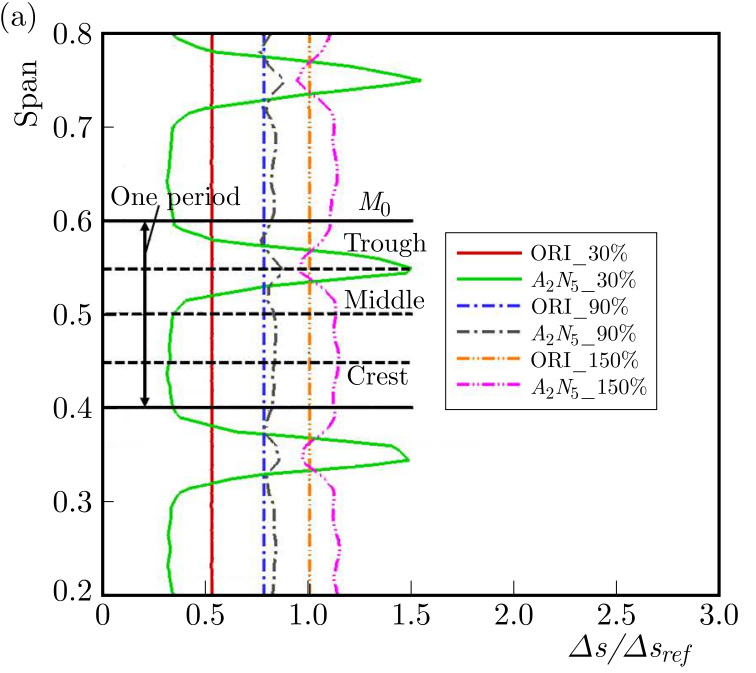

(b)

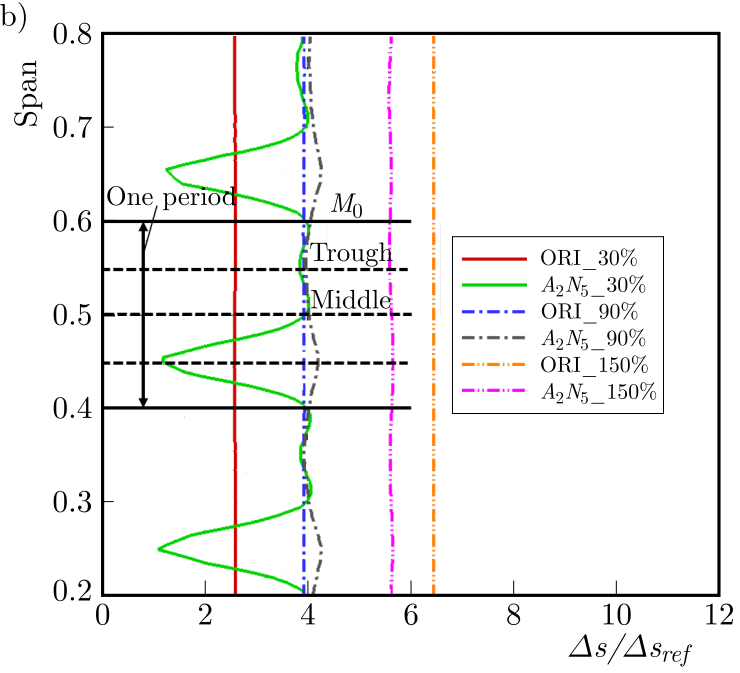

Fig. 7. Spanwise distributions of normalized entropy increase for baseline and $A_{2} N_{5}$ cascades: (a) $i=0^{\circ}$, (b) $i=8^{\circ}$

Streamwise distributions of the normalized entropy increase at several spans for the baseline and $A_{2} N_{5}$ cascades are presented in Fig. 9. For $A_{2} N_{5}$, the $M_{0}$, trough, middle and crest respec- 


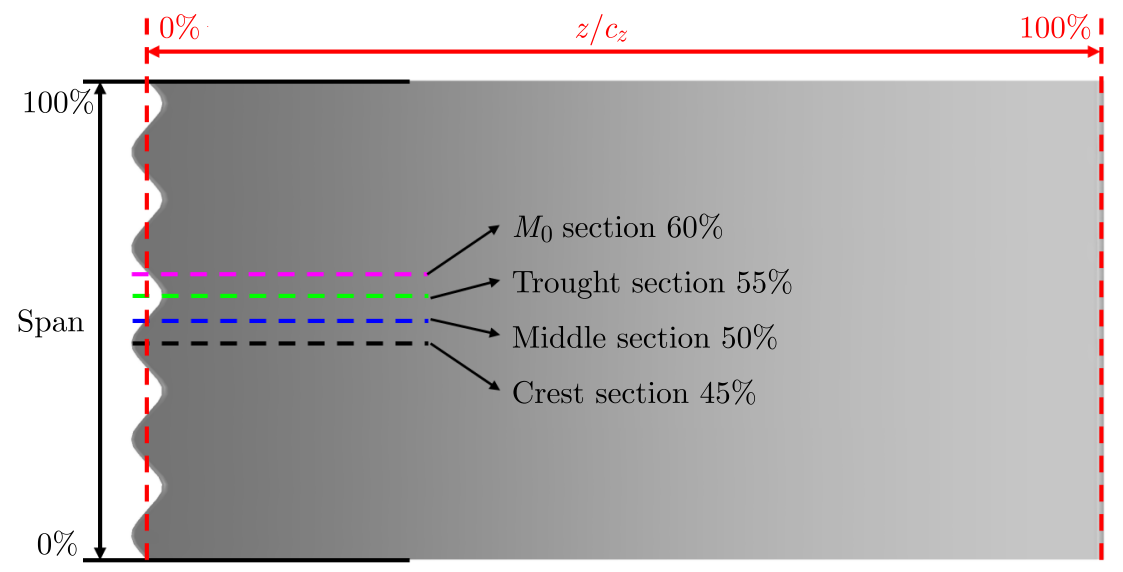

Fig. 8. Locations of $M_{0}$, trough, middle and crest sections

(a)

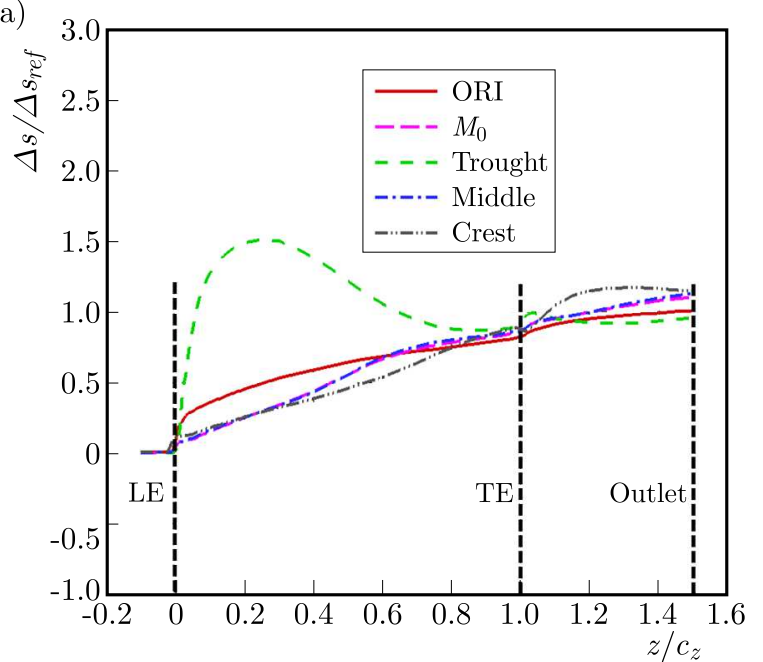

(b)

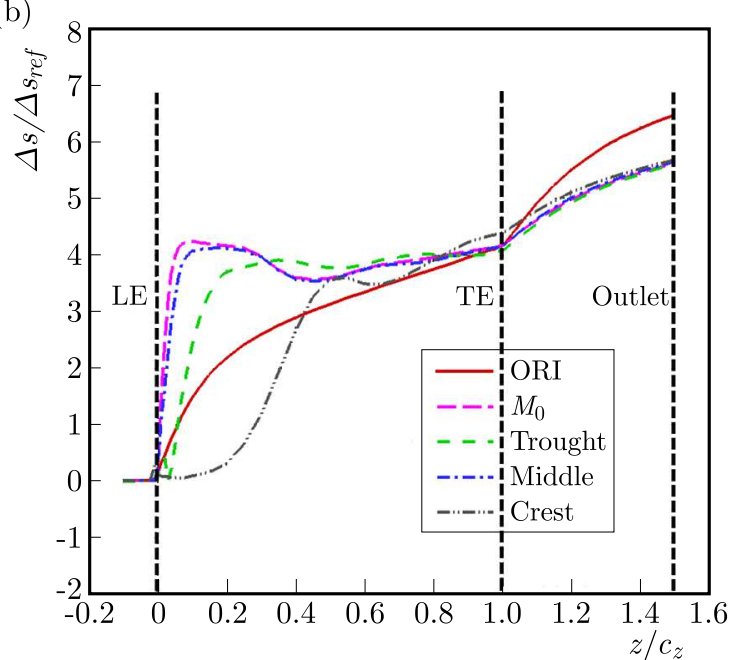

Fig. 9. Streamwise distributions of normalized entropy increase for baseline and $A_{2} N_{5}$ cascades: (a) $i=0^{\circ}$, (b) $i=8^{\circ}$

tively correspond to $60 \%, 55 \%, 50 \%$ and $45 \%$ spans shown in Fig. 8. For the baseline cascade, ORI represents the middle section of $50 \%$ span. Actually, the entropy distributions at these four spans have little difference between one another.

In Fig. 9a, $\Delta s$ of ORI rises gradually downstream LE at $i=0^{\circ}$, and the value of normalized entropy increase equals to 1 at the outlet. As for the $M_{0}$ section, $\Delta s$ also shows a gradual rise downstream LE, and the growing rate is larger than that of ORI. The loss reduction is observed from LE to $60 \% z / c_{z}$ compared to ORI. The $\Delta s$ curve at the middle section is almost the same as that at the $M_{0}$ section. At the trough section, a rapid increase of $\Delta s$ from LE is followed by a decrease till $90 \% z / c_{z}$. The maximum value is reached at $25 \% z / c_{z}$. At the crest section, $\Delta s$ shows the similar trend to that at the $M_{0}$ section despite a slight difference downstream TE. From LE to $80 \% z / c_{z}$, the loss reduction is observed compared to ORI. Among all four sections for $A_{2} N_{5}$, only $\Delta s$ of the trough section is smaller than that of ORI at the outlet.

In Fig. 9b, $\Delta s$ of ORI shows a rapid rise downstream LE at $i=8^{\circ}$, and the value of normalized entropy increase equals to 6.5 at the outlet. At the $M_{0}$ and middle sections, a sharp increase of $\Delta s$ is seen from LE to $20 \% z / c_{z}$, followed by a tender decrease till $46 \% z / c_{z}$ and a subsequent sustained growth till the outlet. As for the trough section, the trend of $\Delta s$ downstream LE is similar to that at the $M_{0}$ and middle sections. A local peak value of the entropy increase lies in the $36 \% z / c_{z}$. At the crest section, an upward trend of $\Delta s$ is observed between LE and $50 \% z / c_{z}$. 
Differently, the loss reduction is achieved from LE to $42 \% z / c_{z}$ at the crest compared to ORI. It is worth nothing that the normalized entropy increases at the $M_{0}$, trough, middle and crest sections come to the same value of 5.6 at the outlet, which is smaller than that for the baseline. The streamwise entropy results are in good consistence with the spanwise distributions of $\Delta s$.

From the above analysis of spanwise and streamwise entropy distributions, it is concluded that the loss reduction at the outlet is merely obtained at the trough section for $A_{2} N_{5}$ in contrast to ORI at $i=0^{\circ}$, while the loss reduction is achieved over the whole span at $i=8^{\circ}$. The introduction of leading edge tubercles turns the uniform spanwise distribution of $\Delta s$ into a nonuniform one and leads to local high loss regions in the fore part of the blade. Also, it is indicated that there exists a spanwise fluid migration downstream LE for $A_{2} N_{5}$, which narrows the gap of $\Delta s$ among the $M_{0}$, trough, middle and crest sections and accounts for the loss decrease from $25 \%$ to $90 \% z / c_{z}$ behind the trough. It is inferred that entropy variations are induced by an altered flow field by leading edge tubercles. The following Section combines the loss development process with flow visualizations, making further efforts to interpret the loss mechanisms.

\subsection{Discussion of loss mechanism}

This Section gives a discussion of the loss development process along the streamwise direction as well as flow visualizations, making further efforts to interpret the loss mechanisms with $A_{2} N_{5}$.

(a)

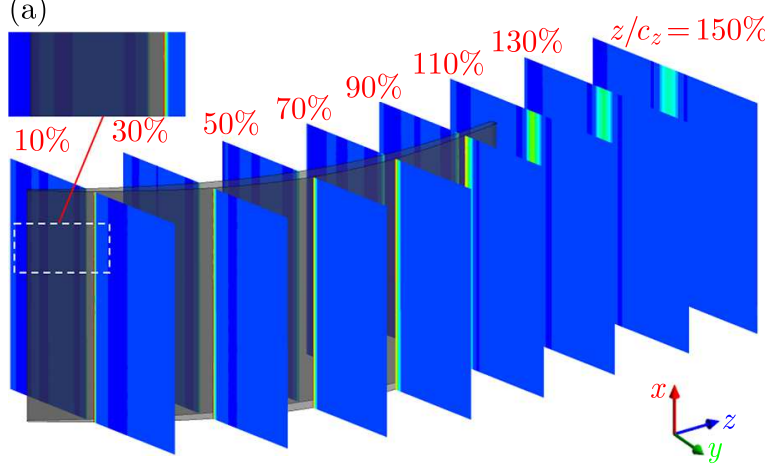

(c)

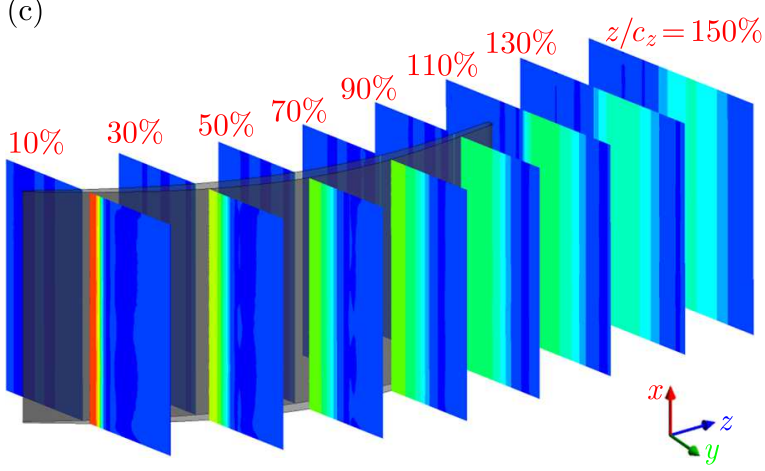

(b)

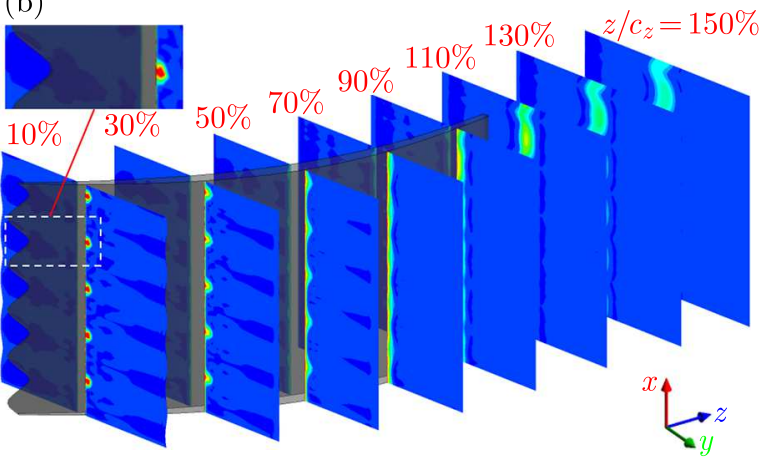

(d)

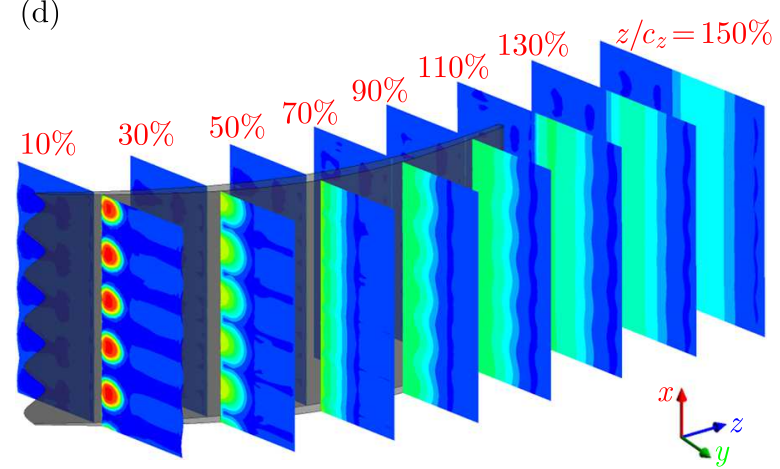

Fig. 10. Distributions of the normalized entropy increase on different axial cross-sections: (a) ORI, $i=0^{\circ}$, (b) $A_{2} N_{5}, i=0^{\circ}$, (c) ORI, $i=8^{\circ}$, (d) $A_{2} N_{5}, i=8^{\circ}$

In Fig. 10, distributions of the normalized entropy increase at $i=0^{\circ}$ and $8^{\circ}$ on different axial cross-sections are shown. The ORI configuration, representing the baseline cascade, shows an almost uniform loss distribution along the blade span in Fig. 10a and 10c. High loss regions are mainly distributed near the blade suction side at $i=8^{\circ}$, see Fig. 10c. From Fig. 10b and 10d, it is observed that the introduction of the leading edge tubercles results in the redistribution of the entropy increase along the span. In the $A_{2} N_{5}$ cascade, high losses accumulate in several 
rounded areas near the blade suction side at $10 \% z / c_{z}$ rather than long narrow strips in the baseline. It is found that high loss regions are in the downstream location of the wave troughs. And the number of high loss regions is equal to the wave number. Without doubt, high loss regions at $i=0^{\circ}$ are much smaller than those at $i=8^{\circ}$. Figure 10d also shows an evidence of the spanwise fluid migration near the blade suction side, which results in the uniform spanwise distribution of $\Delta s$ downstream TE.

Figure 11 shows an isosurface of zero streamwise velocity at $i=8^{\circ}$, which is indicative of the size of the separated region. The baseline case is also presented for comparison. It is apparent from the figure that the size of the recirculation region for $A_{2} N_{5}$ is dramatically reduced, and that separation is delayed relative to the baseline. It can also be seen from the figure that the flow separation is delayed behind crests to a greater extent than behind the troughs. The reduced size of the separation region explains the improved aerodynamic performance. However, separation bubbles are still observed behind the troughs, which correspond with the high loss regions shown in Fig. 10d.

(a)
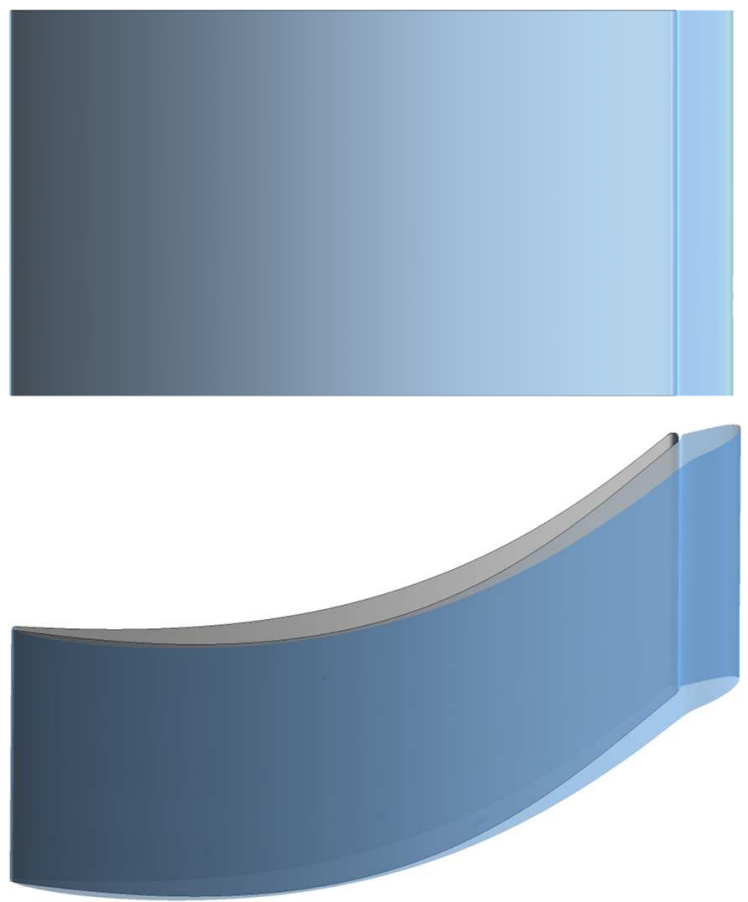

(b)
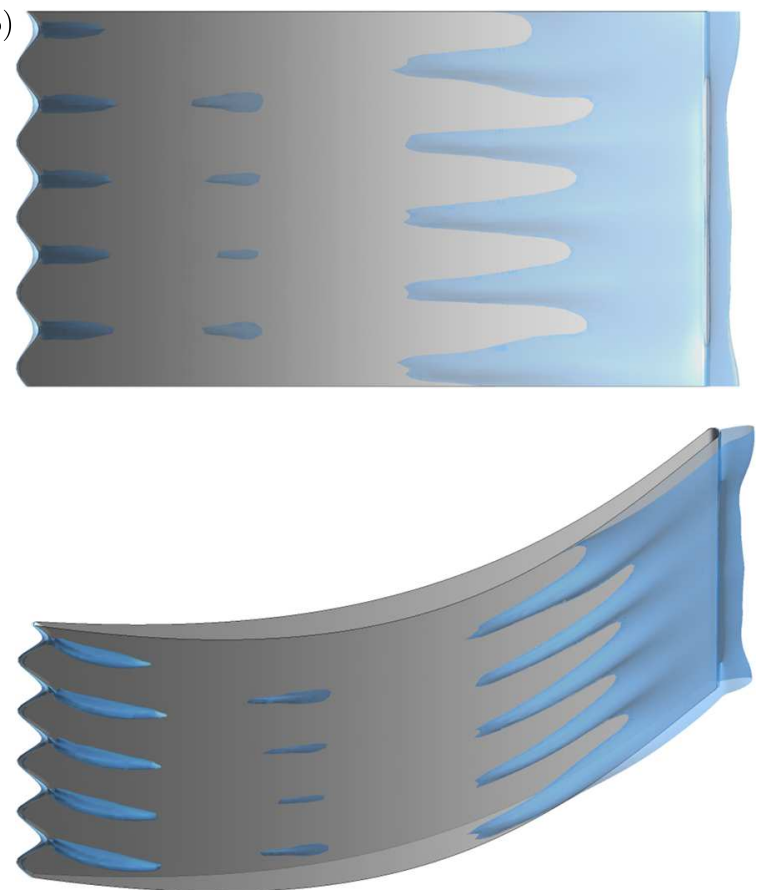

Fig. 11. Isosurface of zero streamwise velocity at $i=8^{\circ}$ : (a) ORI, (b) $A_{2} N_{5}$

Slices of streamwise vorticity plotted in Fig. 12 provide insight into the locality where the normalized entropy increase is greater than 1 at $i=0^{\circ}$ and $8^{\circ}$ and indicate the development of counter-rotating vortices along the streamwise direction.

(a)

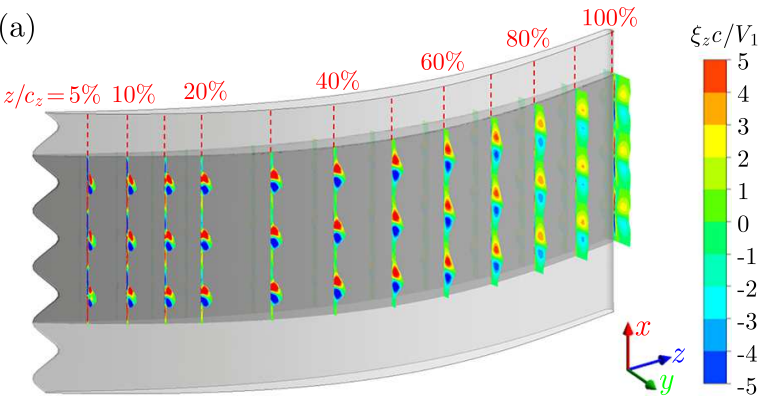

(b)

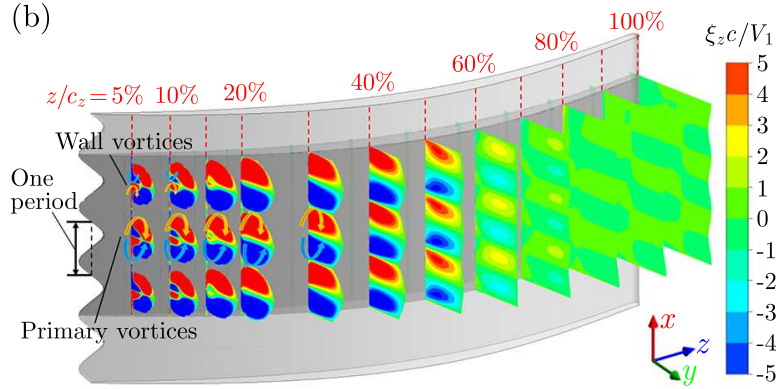

Fig. 12. Slices of streamwise vorticity for $A_{2} N_{5}$ : (a) $i=0^{\circ}$, (b) $i=8^{\circ}$ 
In Fig. 12a, pairs of counter-rotating streamwise vortices are induced by leading edge tubercles and located behind the troughs. The vorticity generally expands in area and declines in strength along the streamlines. And the vortical structure is still visible at $100 \% z / c_{z}$. As a kind of secondary flow, the induced vortices may raise the entropy increase and account for a relatively high loss between LE and TE at the trough section shown in Fig. 9a. It is apparent from Fig. 12b that two different pairs of counter-rotating streamwise vortices are produced behind the wave trough. The wall vortices are adjacent to the primary vortices and have an opposite sense of rotation. The wall vortices gradually disappear along the streamlines and there are only primary vortices visible downstream $15 \% z / c_{z}$. The strength of primary vortices declines along the streamlines and there are almost no vortical structures visible downstream $70 \% z / c_{z}$. It indicates that streamwise vortices feed into the separated flow region and sufficiently interact with flow separation by transfer of the momentum. The interaction significantly delays the flow separation near the blade suction side shown in Fig. 11. It should be noted that there is no flow separation at $i=0^{\circ}$ and this explains the visible vortical structure at $100 \% z / c_{z}$. The wall vortices with primary vortices may be related to the loss increase upstream TE at the trough section and the sharp rise of $\Delta s$ at the $M_{0}$ and middle sections between LE and $20 \% z / c_{z}$ in comparison to the baseline. Another observation that the counter-rotating primary vortices in a pair go away from each other along the streamlines indicates the spanwise movement of vortices, which transports momentum in the spanwise direction.

Figure 13 shows distributions of the pressure coefficient on the suction surface and 3D streamlines at $i=0^{\circ}$ and $i=8^{\circ}$. The pressure coefficient is defined as

$$
C_{p}=\frac{p-\bar{p}_{1}}{\bar{p}_{t 1}-\bar{p}_{1}}
$$

where $\bar{p}_{t 1}$ and $\bar{p}_{1}$ mean the reference total pressure and static pressure in the inlet plane. It is observed in Fig. 13 that the incoming flow is deflected by tubercle geometry such that the bulk of the flow is redirected behind the trough. The deflection results in the spanwise pressure gradient, which transports away the low-momentum fluid near the wall from the crests towards troughs. This pressure gradient also accounts for the production of counter-rotating streamwise vortices.

(a)

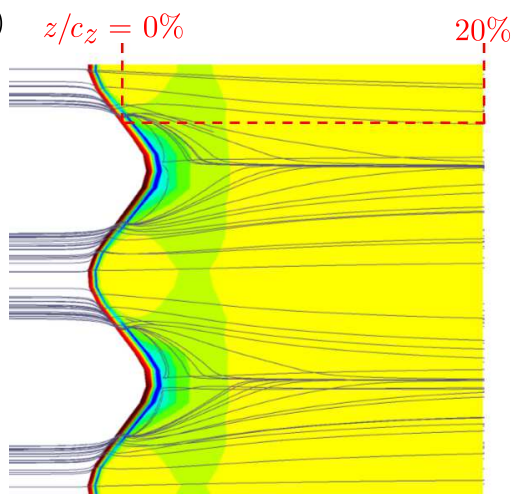

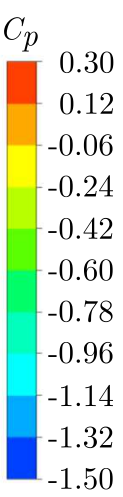

(b)

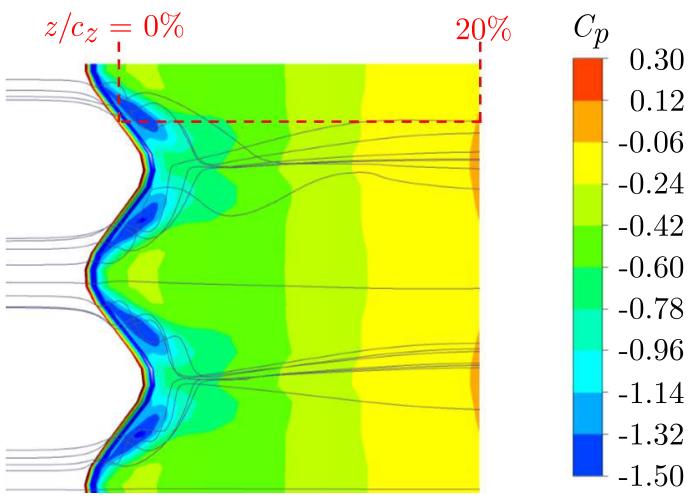

Fig. 13. Distributions of the pressure coefficient on the suction surface and 3D streamlines for $A_{2} N_{5}$ : (a) $i=0^{\circ}$, (b) $i=8^{\circ}$

From the above analysis, loss development procedures along the streamwise direction for ORI and $A_{2} N_{5}$ cascades are clarified. Loss generations for ORI at $i=0^{\circ}$ are primarily ascribed to the viscous boundary-layer near the blade suction side. At a more critical condition of $i=8^{\circ}$, the flow separation and consequent recirculation flow occupies the major part of the high loss. For $A_{2} N_{5}$, the deflection flow resulted from the tubercle geometry leads to the spanwise pressure gradient which transports away the low-momentum fluid near the wall from crests towards the troughs. 
The loss increase at the trough section and loss reduction at the crest section are ascribed to the low-momentum fluid migration in the fore part of the blade. The resulted from the spanwise pressure gradient, two different pairs of counter-rotating streamwise vortices, wall vortices and primary vortices locate behind the troughs and extend to the $M_{0}$ and middle sections in the spanwise direction at $i=8^{\circ}$. The interaction between these vortices and the flow separation by the momentum transfer leads to separation delay and consequent loss reduction at the outlet.

\section{Conclusions}

Taking the lead from humpback whale flippers, leading edge tubercles are applied in a high speed compressor cascade. This paper clarifies the influence of tubercles on loss distributions and elaborate the underlying loss mechanism. Several conclusions are drawn as follows:

- For all configurations of leading edge tubercles investigated in this work, the loss reduction is achieved both at high negative and high positive incidence angles, while a rise in the loss is obtained near the design point. A smaller wave number or a smaller wave amplitude results in lower additional losses at $i=0^{\circ}$, while a larger wave number brings about a greater loss reduction. There is no linear relation between the amplitude and loss at $i=8^{\circ}$.

- Loss generations for ORI at $i=0^{\circ}$ are primarily ascribed to the viscous boundary-layer near the blade suction side. At a more critical condition of $i=8^{\circ}$, the flow separation occupies the major part of the high loss. For $A_{2} N_{5}$, the introduction of leading edge tubercles turns the uniform spanwise loss distribution into a nonuniform one and leads to local high loss regions behind the troughs in the fore part of the blade, both at $i=0^{\circ}$ and $8^{\circ}$. The flow separation is delayed behind the crests to a greater extent than behind the troughs, and the loss reduction is achieved over the whole span in the outlet plane at $i=8^{\circ}$.

- The loss mechanism of tubercle structures by which the size of the flow separation region is reduced has been explored. The tubercle geometry results in the deflection flow and the consequent spanwise pressure gradient. This pressure gradient transports away the low-momentum fluid near the wall from crests towards troughs and also accounts for the formation of counter-rotating streamwise vortices, including wall vortices and primary vortices. The interaction between these vortices and flow separation by the momentum transfer leads to separation delay and consequent loss reduction.

\section{Acknowledgements}

This work has been supported by the National Natural Science Foundation of China (No. 51406115), the "2011 Aero-Engine collaborative Innovation Plan".

\section{References}

1. Denton J.D., 1993, Loss mechanisms in turbomachines, Journal of Turbomachinery, 115, 4, $621-656$

2. Dorfner C., Hergt A., Nicke E., Moenig R., 2011, Advanced nonaxisymmetric endwall contouring for axial compressors by generating an aerodynamic separator-part i: principal cascade design and compressor application, Journal of Turbomachinery, 133, 2, 021026

3. Fischer A., Riess W., Seume J.R., 2003, Performance of strongly bowed stators in a 4-stage high speed compressor, ASME Proceedings, GT2003-38392, 429-435 
4. Fish F.E., Battle J.M., 1995, Hydrodynamic design of the humpback whale flipper, Journal of Morphology, 225, 1, 51-60

5. Fish F.E., Lauder G.V., 2006, Passive and active flow control by swimming fishes and mammals, Annual Review of Fluid Mechanics, 38, 193-224

6. Hansen K.L., Kelso R.M., Dally B.B., 2011, Performance variations of leading-edge tubercles for distinct airfoil profiles, AIAA Journal, 49, 1, 185-194

7. Hergt A., Meyer R., Engel K., 2013, Effects of vortex generator application on the performance of a compressor cascade, Journal of Turbomachinery, 135, 2, 021026

8. Johari H., Henoch C., Custodio D., Levshin A., 2007, Effects of leading-edge protuberances on airfoil performance, AIAA Journal, 45, 11, 2634-2642

9. Keerthi M. C., Kushari A., De A., Kumar A., 2014, Experimental investigation of effects of leading-edge tubercles on compressor cascade performance, ASME Proceedings, GT2014-26242

10. Lord W.K., Macmartin D.G., Tillman T. G., 2000, Flow control opportunities in gas turbine engines, Proceedings of AIAA, AIAA 2000-2234

11. Miklosovic D.S., Murray M.M., Howle L.E., Fish F.E., 2004, Leading-edge tubercles delay stall on humpback whale (Megaptera novaeangliae) flippers, Physics of Fluids, 16, 5, L39-L42

12. Pedro H.T.C., Kobayashi M.H., 2008, Numerical study of stall delay on humpback whale flippers, Proceedings of AIAA, AIAA 2008-0584

13. Steinert W., Eisenberg B., Starken H., 1991, Design and testing of a controlled diffusion airfoil cascade for industrial axial flow compressor application, Journal of Turbomachinery, 113, 4, 583-590 